\title{
Survey Of GNSS Coordinates Systems
}

\author{
Martina Szabova, M.Sc., \\ Frantisek Duchon, Associate Prof., M.Sc. PhD, \\ Slovak University of Technology in Bratislava, Slovakia
}

doi: 10.19044/esj.2016.v12n24p33 URL:http://dx.doi.org/10.19044/esj.2016.v12n24p33

\begin{abstract}
The use of satellite positioning systems to determine position in reference frame can introduce serious practical difficulties. The problem can be in the fields of navigation, map revision or cadastral surveying. It arises because in local area the local coordinate system were used. The problem can be solved by transformation between coordinates frame. Global navigation satellite systems (GNSS) don't use same reference frame and it is important to know transformation between this systems. This paper works with information of many international organizations and their documents. It contains information about reference coordinate system of GNSS, information about local coordinates system used in North America, UK, and Europe.
\end{abstract}

Keywords: Reference frames, coordinates, coordinate reference systems, local coordinate systems

\section{Introduction}

Satellites coordinates and user's coordinates must be defined in known reference system. Precise positioning by GNSS requires an accurate definition and determination of this reference system. There are two main reference system used in GNSS navigation: Conventional Celestial Reference System (CRS) and Conventional Terrestrial Reference system (TRS).

CRS is an inertial reference system. The origin of this system is in the Earth's center of the mass. The $\mathrm{X}$ axis heading to equinox, the $\mathrm{Z}$ axis is orthogonal to equatorial, and the $\mathrm{Y}$ axis is complementing the right-handed system.

TRS is system, which is co-rotating with the Earth. It is also called Earth-Centered, Earth-Fixed system (ECEF). The origin of this system is same as in CRS, in the Earth's center of the mass. The X axis is heading to intersection of the equatorial and mean Greenwich meridian, the $\mathrm{Z}$ axis is 
same as axis of Earth's rotation and it is heading to Conventional Terrestrial Pole (CTP), and the Y axis is complementing the right-handed system. In figure 1 CRS is shown and in figure 2 TRS is shown.

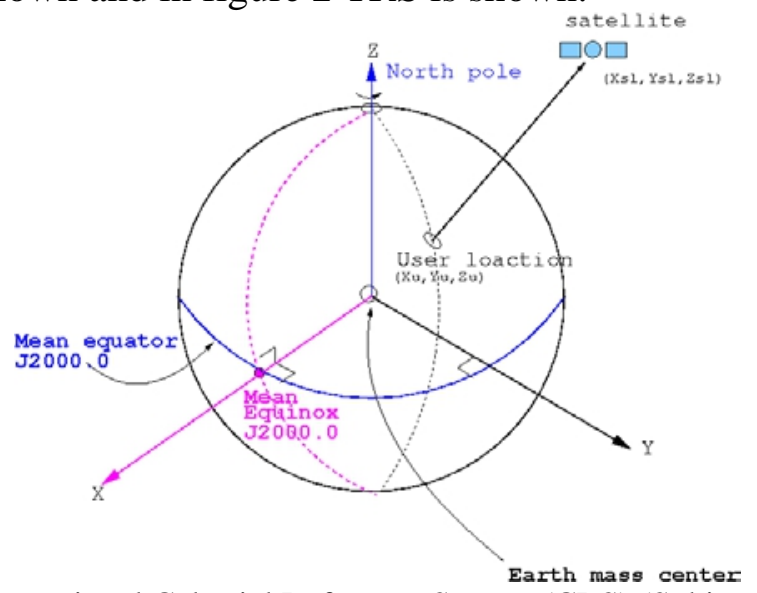

Figure 1: Conventional Celestial Reference System (CRS) (Subirana, 2013)

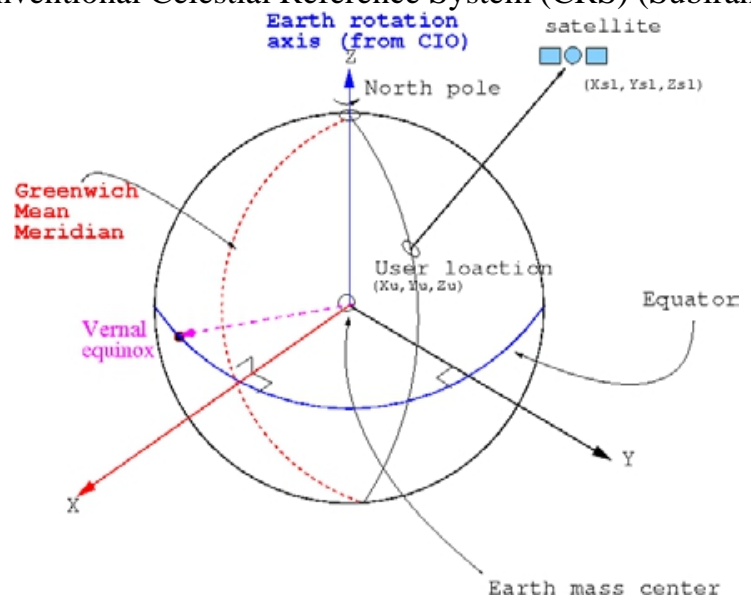

Figure 2: Conventional Terrestrial Reference System (TRS) (Subirana, 2013)

International Terrestrial Reference Frame (ITRF) is TRS. ITRF provides a set of coordinates of points located on the Earth. It can be used to measure tectonics plate, regional subsidence. The Earth Orientation Parameters (EOP) connect the TRS and CRS. The four geodetic techniques are used for computation accurate coordinates: GPS, VLBI, SLR, and DORIS. As the period of data available increases with time the ITRF is updated. From 1988 ITRF has 11 different realizations, the latest one is ITRF2005. All these realizations include station positions and velocities. (Subirana, 2013) (Hofmann-Wellenhof, 2008) (Misra, 2011) (Grafarend, 1995) 


\section{Geodetic Datum in General}

The three-dimensional position requires a three dimensional surface for depiction. A suitable surface, which is representing the Earth, is the equipotential surface of the Earth's gravity field called geoid. Due to variations in gravity, the geoid has no regular mathematical representation. A good mathematical model is an ellipsoid or spheroid. Global datum is the best fitting regular surface on whole geoid. Local datum best fits in a local area. A simple example of the spatial relationship between the geoid, a global datum and a local datum is in figure 3.

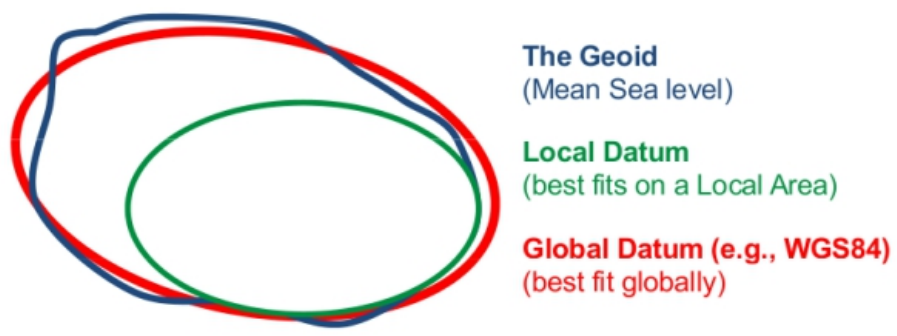

Figure 3: Geoid and Global and Local geodetic datums. (Subirana, 2013)

The development of space based measurement system requires single global datum instead of geodetic local datum. The space based measurement techniques have some fundamental influences on datum definition:

- $\quad$ Satellites move around the center of mass of the earth and require a datum which is geocentric.

- Their global nature has meant that what has previously been considered geodetic science is having increasing importance in day to day surveying.

- $\quad$ Height from these systems is measured above the ellipsoid which has required better geoid models.

- $\quad$ There has been a trend to revise local working datum to be more compatible with measurements from systems such as GPS, GLONASS, and Galileo.

- $\quad$ Their three dimensional nature has led to a need to closely relate horizontal and vertical datum.

A global datum is based on the Conventional Terrestrial Reference System (TRS). Particularly realizations of the CTRS are World geodetic system WGS 84 (GPS), PZ-90 (Glonass), GTRS (Galileo) and International Terrestrial Reference Frame (ITRF), which is not established by military organizations, but by a scientific institution.

A local datum is typically used for civil and military applications of one country or small groups of countries. In the local datum the ellipsoid is defined as the best fit to the geoid in region of interest. It is not necessary to 
be a geocentric as the global datum. In many local applications the ellipsoidal coordinates must be transformed from surface to the plane.

The coordinates in one datum can be transformed into another datum with a 7 parameter transformation, Transformation between Celestial and Terrestrial Frames. The most commercial receivers can have output in several official datums. (Subirana, 2013) (Hofmann-Wellenhof, 2008) (Grafarend, 1995) (Zhiping, 2014) (Chang, 2013)

\section{World Geodetic System 1984}

World Geodetic System 1984 (WGS 84) is a coordinate system, which is standard for use in Geodesy, Cartography and navigation by GPS. WGS 84 is a realization of the conventional terrestrial reference system. WGS 84 is reference frame for GPS system. The main reference document is the NIMA Technical Report on WGS 84.

WGS84 can refer to two types of coordinates: WGS $84 \mathrm{XYZ}$ coordinates and WGS 84 LLA coordinates (Longitude, Latitude, and Altitude), which are called geodetic coordinates.

The coordinate origin is placed in the center of mass of the Earth with maximum error $2 \mathrm{~cm}$. Meridian of zero longitude is the IERS reference meridian, which is $102.5 \mathrm{~m}$ east from Greenwich meridian. Axis $\mathrm{X}$ is heading to intersection of equatorial and zero meridian. Axis $\mathrm{Z}$ is heading to the Conventional Terrestrial Pole (CTP). Axis Y is complementing righthanded coordinate system. In figure 4 is shown WGS 84 coordinate system.

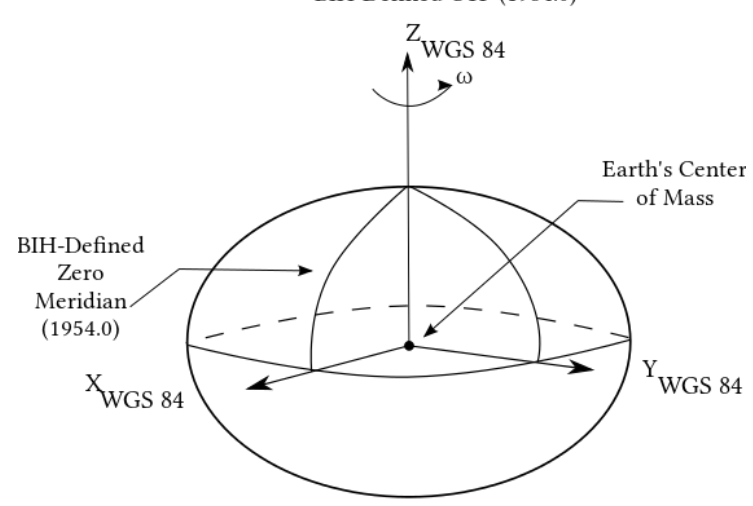

Figure 4: WGS84 reference frame (Subirana, 2013)

The fundamental parameters of WGS 84 are in table 1.

\begin{tabular}{|c|c|}
\hline Parameter & Value \\
\hline Ellipsoid - Semi-major axis (a) & $6378137.0 \mathrm{~m}$ \\
\hline Ellipsoid - Reciprocal flattening (1/f) & 298.257223563 \\
\hline Earth's angular velocity $\left(\omega_{\mathrm{E}}\right)$ & $7292115 \mathrm{E}-11 \mathrm{rad} / \mathrm{sec}$ \\
\hline Earth's gravitational constant $(\mathrm{GM})$ & $3986004.418 \mathrm{E} 8 \mathrm{~m}^{3} / \mathrm{s}^{2}$ \\
\hline
\end{tabular}

Table 1: The fundamental parameters of WGS 84 (Misra, 2011) 
This coordinate system is most used in application. Commonly used GNSS receivers has output in WGS 84 LLA, which are in degrees. This is big advantage of this system. (Hofmann-Wellenhof, 2008) (Misra, 2011) (Stanaway, 2005) (Fell, 2001)

\section{Glonass Reference Frame PZ-90}

The Glonass satellites broadcast ephemerides, which are in the PZ-90 reference frame. PZ-90 coordinate system is type of ECEF (Earth-Centered Earth-Fixed).

The transformation between PZ-90 $(x, y, z)$ and WGS $84\left(x^{\prime}, y^{\prime}, z^{\prime}\right)$ is determined to meter level accuracy by following equation:

$$
\left[\begin{array}{l}
x^{\prime} \\
y^{\prime} \\
z^{\prime}
\end{array}\right]=\left[\begin{array}{l}
x \\
y \\
z
\end{array}\right]+\left[\begin{array}{ccc}
-3 \text { ppb } & -353 \text { mas } & -4 \text { mas } \\
353 \text { mas } & -3 \text { ppb } & 19 \text { mas } \\
4 \text { mas } & -19 \text { mas } & -3 p p b
\end{array}\right]\left[\begin{array}{l}
x \\
y \\
z
\end{array}\right]+\left[\begin{array}{c}
0.07 \mathrm{~m} \\
-0.0 \mathrm{~m} \\
-0.77 \mathrm{~m}
\end{array}\right]
$$

Where $p p b$ is parts per billion $\left(1 p p b=10^{-9}\right)$ and mas is milliarcseconds $\left(1\right.$ mas $\left.=4.84813681 .10^{-9} \mathrm{rad}\right)$.

The fundamental parameters of system PZ-90 is in table 2:

\begin{tabular}{|c|c|c|}
\hline Parameter & WGS 84 & PZ-90 \\
\hline Ellipsoid - Semi-major axis (a) & $6378137.0 \mathrm{~m}$ & $6378136.0 \mathrm{~m}$ \\
\hline Ellipsoid - Reciprocal flattening (1/f) & 298.257223563 & 298.257839303 \\
\hline Earth's angular velocity $\left(\omega_{\mathrm{E}}\right)$ & $7292115 \mathrm{E}-11 \mathrm{rad} / \mathrm{s}$ & $7292115 \mathrm{E}-11 \mathrm{rad} / \mathrm{s}$ \\
\hline Earth's gravitational constant $(\mathrm{GM})$ & $3986004.418 \mathrm{E} 8 \mathrm{~m}^{3} / \mathrm{s}^{2}$ & $3986004.4 \mathrm{E} 8 \mathrm{~m}^{3} / \mathrm{s}^{2}$ \\
\hline
\end{tabular}

Table 2: The fundamental parameters of WGS 84 and PZ-90 (Hofmann-Wellenhof, 2008)

Glonass modernization plan implements the new reference system PZ-90.02 in emphemeris information on 20 September 2007. This reference system is updated version of PZ-90, which is the closet one to system ITRF2000 (Inertial Terrestrial Reference Frame). The transformation between them contains only one shift vector of origin and no rotations or scale vector. (Hofmann-Wellenhof, 2008) (Pick, 1985) (Zhiping, 2014) (Chang, 2013)

\section{Galileo Reference Frame - GTRF}

The reference frame of Galileo shall be an independent realization of ITRF. The requirement for Galileo terrestrial reference frame (GTRF) is that the difference between latest version of ITRF and GTRF must be less than 3 $\mathrm{cm}(2 \sigma)$. Connection to the ITRF is validated by International GNSS Stations (IGS). IGS are part of the ITRF system.

The initial coordinates of reference stations were provided by GPS observations. Subsequent GTRF versions will use both Galileo and GPS observations. (Hofmann-Wellenhof, 2008) (Pick, 1985) (Zhiping, 2014) (Chang, 2013) 


\section{European Terrestrial Reference System}

The European Terrestrial Reference System 1989 (ETRS89) is Cartesian reference frame, which is ECEF. Coordinates of place in Europe in ETRS89 are not subject to change due to continental drift. ETRS89 is based on ITRS at the epoch 1989.0. Due to the continental drift difference between ETRS89 and ITRS is around $2.5 \mathrm{~cm}$ per year.

The ETRS has some frame realizations, which are labeled by year (e.g. ETRF2000) means that the system is the same as ITRS in that time. Transformation between ETRS89 and ITRS is time independent. ETRS89 is same as NAD83 in North America. It is based on reference ellipsoid GRS80. (Hofmann-Wellenhof, 2008) (Pick, 1985) (Zhiping, 2014) (Chang, 2013)

\section{East-North-Up (ENU) frame}

The East-North-UP (ENU) reference frame is a local frame, which is centered in the navigation system origin. $\mathrm{Z}(\mathrm{U})$ axis is heading upward along the ellipsoidal normal, $\mathrm{X}(\mathrm{E})$ axis is pointing toward geodetic East and $\mathrm{Y}(\mathrm{N})$ axis is pointing toward geodetic North. The Transformation between ECEF and ENU includes the shift and two rotations.

From ENU to ECEF frame the first rotation is clockwise rotation over East (X) axis by an angle $90-\varphi$. This rotation aligns Up (Z) axis with $\mathrm{Z}_{\mathrm{ECEF}}$ axis. Second rotation is a clockwise rotation over the $\mathrm{z}$-axis by an angle $90+\lambda$. This rotation aligns East $(\mathrm{X})$ axis with the $\mathrm{X}_{\mathrm{ECEF}}$ axis.

$$
\left(\begin{array}{l}
\Delta x \\
\Delta y \\
\Delta z
\end{array}\right)=R_{z}\left[-\left(\frac{\pi}{2}+\lambda\right)\right] R_{E}\left[-\left(\frac{\pi}{2}-\varphi\right)\right]\left(\begin{array}{l}
\Delta e \\
\Delta n \\
\Delta u
\end{array}\right)
$$

Where $\varphi$ and $\lambda$ are ellipsoidal coordinates of the origin of the ENU frame. ENU frame is shown in figure 5. (Subirana, 2013) (Misra, 2011) (Dawson, 2005) (Grooves, 2013)

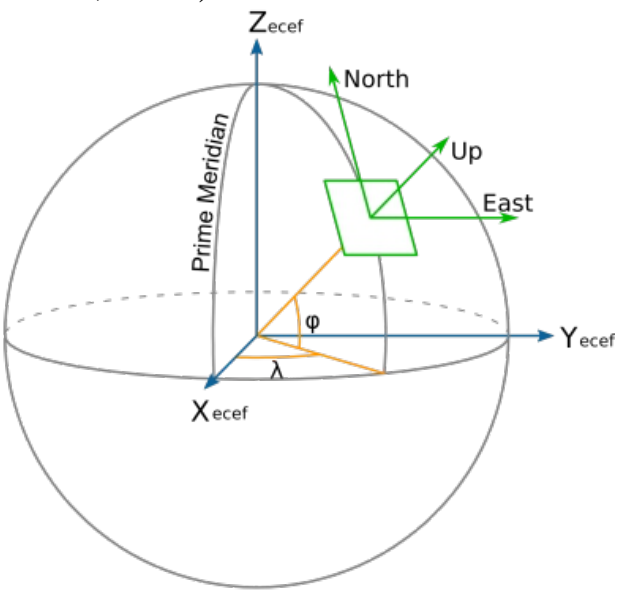

Figure 5: ENU frame (Misra, 2011) 


\section{North American Datum (NAD)}

North American Datum (NAD) is used in North America, and it defines geodetic network in North America. Two types of NAD are used the North American Datum of 1927 (NAD27) and the North American Datum of 1983 (NAD83).

NAD27 is based on surveys of the entire continent. Measurements were based on a triangulation station, which is in intersection of triangulation arc on 39th parallel north and triangulation arc along the 98th meridian west. NAD27 is oriented by the azimuth from Meades Ranch Triangulation Station to Waldo. NAD27 is based on the Clarke 1866 Ellipsoid with semi-major axis $6378206.4 \mathrm{~m}$, semi-minor axis $6356583.8 \mathrm{~m}$, and calculated inverse flattening is 294.978698214. The latitude and the longitude in North America are based on distance and direction from Meades Ranch. NAD27 is older and is used in many topographic maps and many major projects by the United States Army Corps of Engineers.

In 1983 NAD83 was defined. NAD83 is based on another ellipsoid GRS 80 with semi-major axis $6378137 \mathrm{~m}$, semi-minor axis $6356752.3141 \mathrm{~m}$, and calculated inverse flattening 298.257222101. The GRS 80 ellipsoid approximates the Earth as a whole. Identical coordinates in NAD27 and NAD83 can be for different place in North America. NAD27 is defined by coordinates of initial point Meades Ranch and NAD83 is Earth-centered and it has no initial point or direction. Shift between NAD27 and NAD83 is in figure 6.

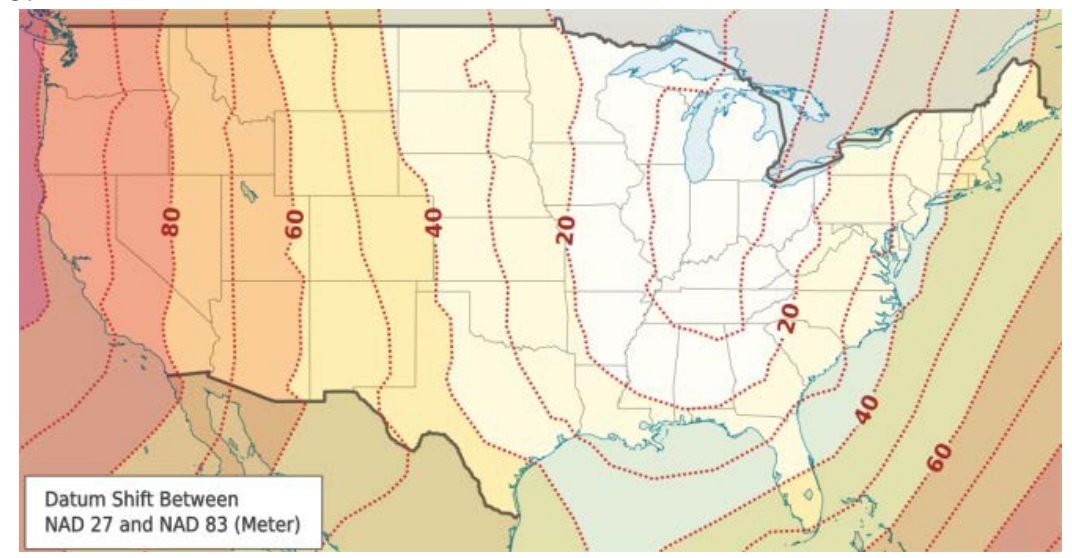

Figure 6: Datum shift between NAD27 and NAD83 in meters (Zhiping, 2014)

The fundamental difference between NAD83 and WGS 84 is that NAD83 is defined to remain constant over time for points on the North American Plate. WGS 84 is defined with respect to the stations all over the world. Difference is around 1-2 cm per year. (Misra, 2011) (Chang, 2013) (Zhiping, 2014) 


\section{European Datum 1950}

European Datum 1950 (ED50) is datum, which was defined for the international connection of geodetic networks in Western Europe.

ED50 is based on International Ellipsoid 1924 (Hayford-Ellipsoid). This ellipsoid was mostly used until ellipsoid GRS80 was defined. ED50 is centered at Helmertturm in Potsdam. ED50 is part of the fundamentals of the NATO coordinates (Gauss-Krüger and Universal Transverse Mercator UTM). (Zhiping, 2014)

\section{Universal Transverse Mercator (UTM)}

UTM is conformal projection, which uses a two-dimensional Cartesian coordinate system - horizontal position representation. UTM is a multiple map projection. The Earth is divides into sixty zones. Each zone is $8^{\circ}$ wide and $6^{\circ}$ long. Nowadays UTM is based on ellipsoid WGS 84.

In each zone the scale factor reduce the diameter of the transverse cylinder to produce a secant projection with two standard lines. The scale is less than 1 inside and greater than 1 outside the standard lines. The overall distortion is minimized. Scale increases in boundaries between two UTM zones. In figure 7 the zone map of Europe is shown.

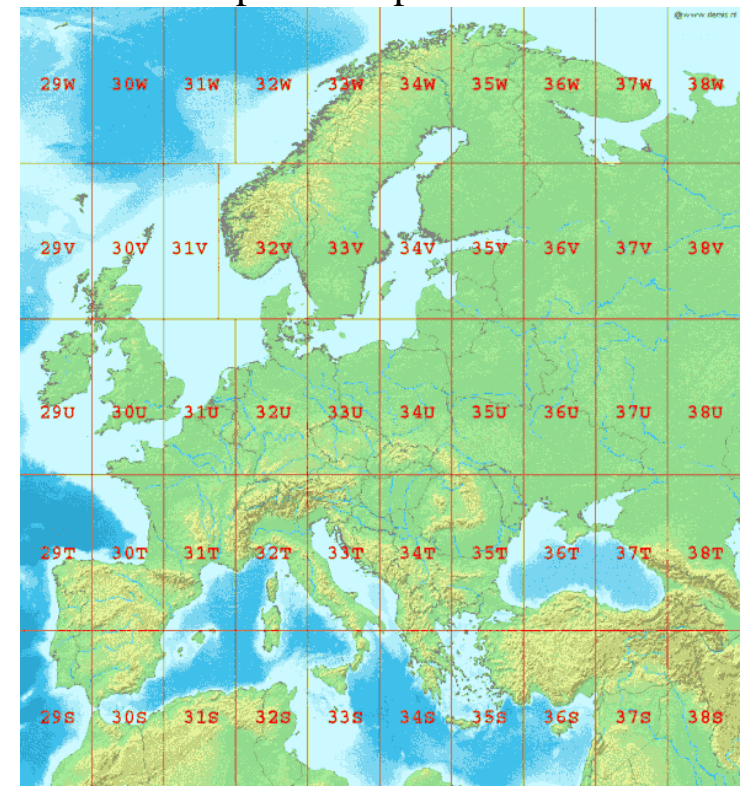

Figure 7: UTM Zones for Europe (Karney, 2011)

The origin for every zone is in the intersection of the zone's central meridian and the equator. The central meridian is shift $500000 \mathrm{~m}$ east, because of avoiding dealing with negative numbers. Number of each zone consist of number (1-60) and letter (C-X). Number describes in which 
vertical line the zone is and the letter describes in which horizontal line the zone is. (Zhiping, 2014) (Chang, 2013) (Grafarend, 1995)

\section{Conclusion}

Each of Global navigation satellite systems has own reference coordinates system, which has known transformation equation to the coordinate system of ITRS. Position measured by GNSS is mostly in WGS84 coordinates. ETRS89 is ITRS system tied to the stable part of the European plate in 1989. It difference growing by about $2.5 \mathrm{~cm}$ per year.

Local coordinates systems are mostly used in cadaster, mapping and navigation. The ENU system is commonly used in robotics navigation. In cadaster there are used local reference frames as NAD83 for North America or ED50 for Western Europe.

\section{Acknowledgement}

The authors would like to thank the Ministry of Education, Science, Research and Sport of the Slovak Republic for funding project VEGA $1 / 0065 / 16$, and the Slovak research and development agency for funding project APVV-14-0894.

\section{References:}

Subirana, J.S., Zornoza, J.M.J., \& Hernándes-Pajares, M. (2013). GNSS Data Processing, Vol I: Fundamentals and Algorithms. Noordwijk: ESA Communications

Hofmann-Wellenhof, B., Lichtenegger, H., \& Wasle, E. (2008). GNSS Global Navigation Satellite Systems. Wien: Springer

Misra, P. \& Enge, P. (2011). Global Positioning System: Signals, Measurements, and Performance. Austin: Ganga-Jamuna Press

Grafarend, E. (1995). Geodetic Theory Today. Berlin: Springer

Gleason, S. \& Gebre-Egziabher, D. (2009). GNSS Application and Methods. Boston: Artech House

Grooves, P.D. (2013). Principles of GNSS, Inertial, and Multisensor Integrated Navigation Systems. Boston: Artech House

Zhiping, L., Yunying, Q., \& Shubo, Q. (2014). Geodesy - Introduction to Geodetic Datum and Geodetic Systems. Berlin: Springer

Fell, F. \& Tanenbaum, M. (2001). Preliminary comparisons of the WGS84(EGM 96) geoid with national vertical datums. Honolulu, HI: OCEANS, 2001. MTS/IEEE Conference and Exhibition

Stanaway, R. \& Dawson, J. (2005). The Divorce of Two Datums. Honolulu, HI: Position Magazine Nr. 20

Stanaway, R. (2005). GDA94, ITRF, WGS84: WHAT'S THE DIFFERENCE? WORKING WITH DYNAMIC DATUMS. Carlton 
Chang, K.-T. (2013). Introduction to Geographic information systems. New York, NY: McGraw-Hill Higher Education

Pick, M. (1985). Closed formulae for transformation of the cartesian coordinate system into a system of geodetic coordinates. Studia Geophysica et Geodaetica. Netherlands: Springer

Karney, C.F.F. (2011). Transverse Mercator with an accuracy of a few nanometer. Journal of Geodesy. Springer-Verlag 